\section{Elévation de la tension artérielle lors de chirurgie sous garrot pneumatique: un mécanisme indépen- dant de la rénine?}

Le but de ce travail a été d"évaluer l'efficacité du captopril dans la prévention de lélévation de la pression artérielle lors de chirurgie du membre inférieur sous garrot pneumatique.en anesthésie balancée. Vingt patients consécutifs ont été inclus dans cette étude et randomisés en deux groupes. Lun ( $n=$ 10), recevant un comprimé de captopril de $50 \mathrm{mg}$ avec la prémédication, l'autre $(n=10)$, recevant un comprimé de placebo au même moment. Les différentes variables étudiées (tension artérielle, fréquence cardiaque) ont été mesurées continuellement. Cette étude a démontré, que le prétraitement par du captopril ne permet pas de prévenir une augmentation de la pression artérielle pendant la durée de gonflage d'un garrot pneumatique, les moyennes des tensions artérielles systoliques et diastoliques, à la fin de la période de gonflage du garrot, sélèvent a 128/86 et 128/81 dans le groupe captopril et dans le groupe placebo, respectivement. Ce résultat montre que le système rénine-angiotensine nïntervient pas, ou que peu, dans le phénomène d'augmentation des valeurs tensionnelles induite par un garrot pneumatique.

The aim of this study was to evaluate the effectiveness of captopril for the prevention of the increase of arterial pressure during orthopaedic surgery requiring the application of lower limb tourniquets with balanced anaesthesia. Twenty consecutive patients were included in the study and were randomly divided into two groups. The first $(n=10)$ received $50 \mathrm{mg}$ captopril

\section{Key words}

ANAESTHETIC TECHNIQUE: balanced; HORMONES: renin, angiotensin; PHARMACOLOGY: captopril.

*Service d'Anesthésiologie, Centre Hospitalier Universitaire Vaudois, 1011 Lausanne, $\mathrm{CH}$. † Service d'Anesthésiologie, Hôpital de zone, 1110 Morges, $\mathrm{CH}$.

Correspondance à adresser. L. Magnusson, Service

d'Anesthésiologie, CHUV, 1011 Lausanne, CH.

Accepté pour publication le 14 mars, 1993.
Lennart Magnusson MD, ${ }^{*}$ Mino Rebagliati MD, $\dagger$ Eric Buchser MD† orally together with the preanaesthetic medication, the second $(n=10)$ received a placebo at the same time. The different variables studied (arterial pressure, heart rate) were continuously measured. This study demonstrated that the pretreatment with captopril did not prevent an increase of the arterial pressure during the application of a tourniquet. The means of the systolic and diastolic arterial pressures at the end of the application of the tourniquet were 128/86 and 128/81 in the captopril group and the placebo group, respectively. This result shows that the renin-angiotensin system does not significantly contribute to the increase of the arterial pressure induced by a tourniquet.

L'utilisation d'un garrot pneumatique pour la chirurgie du membre inférieur peut entraîner une élévation de la pression artérielle (EPA) voire une hypertension artérielle systémique (HTA), sans tachycardie associée. Celle-ci débute en général 30 à 45 minutes après le gonflage du garrot, elle cède rapidement après le relâchement du garrot. ${ }^{1-4}$ Cette EPA est très fréquente $(67 \%$ des cas) lors d'intervention en anesthésie générale, alors qu'elle est rare (2,7\% des cas) sous anesthésie spinale. ${ }^{4-5}$

Les mécanismes précis responsables de cette EPA restent mal connus, de même que les médiateurs qui la provoquent. Toutefois, des études éléctrophysiologiques ont montré que l'anesthésie générale ninhibe pas les fibres nociceptives $A$ delta et $C$, lesquelles transmettent des informations nociceptives au système nerveux central. Dès lors, ces afférences pourraient induire, par l'intermédiaire du système nerveux sympathique, une réponse hypertensive systémique. ${ }^{3,6,7}$

Une étude récente, chez 20 patients opérés des membres inférieurs sous anesthésie générale et avec mise en place d'un garrot pneumatique, ${ }^{8}$ montre que le développement d'une EPA est associée à une hyperactivité du système nerveux sympathique, documentée par une élévation significative des concentrations plasmatiques de l'adréna- 
line, de la noradrénaline, du facteur atrial natriurétique et de l'activité de la rénine (ARP). L'administration prophylactique de clonidine a significativement atténué cette réponse sympathique, sans toutefois l'abolir. L'apparition de cette EPA n'est donc pas encore totalement expliquée et un rôle de l'axe rénine-angiotensine demeure possible. Le but de cette étude a donc été de préciser le rôle de la rénine dans ce contexte en évaluant l'efficacité de l'administration prophylactique d'une dose de captopril sur l'apparition de cette EPA.

Parmi les inhibiteurs de l'enzyme de conversion, le captopril a été choisi en raison de ses propriétés pharmacocinétiques. La résorption est rapide, le début d'action apparaît en 15-20 minutes et les concentrations plasmatiques maximales sont atteintes entre 30 et 90 minutes après l'absorption. ${ }^{9}$ L'administration d'un inhibiteur de l'enzyme de conversion a pour conséquence l'augmentation de l'activité de la rénine plasmatique (ARP), notamment en raison d'une inhibition du rétrocontrôle négatif que l'AII exerce sur la sécrétion de rénine. Cet effet, qui témoigne indirectement de l'efficacité de l'inhibiteur, s'observe même aux faibles doses. Chez 27 patients, $25 \mathrm{mg}$ de captopril ont significativement augmentées l'ARP. ${ }^{10}$ Une dose de $50 \mathrm{mg}$ de captopril per os a été arbitrairement choisie, laquelle garantit une inhibition suffisante de l'enzyme de conversion pour que l'ARP augmente.

\section{Patients et méthodologie}

\section{Patients}

Après acceptation par le Comité d'éthique de notre institution, 20 patients consécutifs, normotendus, âgés de 20 à 65 ans, ASA I ou II, programmés pour une chirurgie orthopédique du genou nécessitant l'utilisation d'un garrot pneumatique ont été inclus dans cet essai. Chaque patient a au préalable donné son consentement oral. Les femmes enceintes ont été exclues, de même que les patients présentant une surcharge pondérale de plus de $20 \%$ ou une atteinte pulmonaire, cardiaque, rénale, hépatique, neurologique, une maladie métabolique instable (en particulier un diabète) ou des antécédents d'allergies graves. Ont également été exclus, les patients traités par des antiinflammatoires non-stéroïdiens durant la semaine précédant l'intervention (diminution de l'effet anti-hypertenseur des inhibiteurs de l'enzyme de conversion), ainsi que ceux ayant reçu des médicaments antinéoplasique, des stéroïdes, de l'allopurinol ou du procaïnamide (risque de leucopénie). ${ }^{9,11}$

\section{Méthode}

Les comprimés de captopril (Lopirin) ${ }^{\circledR}$ ainsi que ceux de placebo, ont été fournis par la maison Squibb SA. Ils ont été répartis par la pharmacie de l'hôpital en 20 sachets identiques, contenant soit 1 comprimé de captopril à $50 \mathrm{mg}(n=10)$, soit 1 comprimé placebo $(n=10)$. La randomisation a été effectuée par tirage au sort.

Vingt-quatre heures avant l'intervention, l'anesthésiste responsable de l'étude (L.M.) a rendu visite aux patients pour expliquer les buts de linvestigation et obtenir leur consentement. Une heure avant l'induction de l'anesthésie, on a administré une prémédication par voie intramusculaire, comprenant du midazolam 5 à $7,5 \mathrm{mg}$ associé à de l'atropine $0,5 \mathrm{mg}$. Les patients ont également reçu soit 1 comprimé de $50 \mathrm{mg}$ de captopril, soit 1 comprimé de placebo. Quinze minutes plus tard, les patients ont été transférés en salle de réveil et placés sous surveillance électrocardioscopique. La pression artérielle a été mesurée de manière non invasive au moyen d'une manchette de pression automatique effectuant une mesure à intervalle de cinq minutes (Blut-druck Monitor ${ }^{\circledR}$ BP-103N Hoyer-Bremen).

La technique d'anesthésie a été standardisée: après avoir mis en place une voie veineuse périphérique de 19G, $500 \mathrm{ml}$ de Ringer-Lactate ont été perfusés en 30 minutes. L'induction, précédée par trois minutes de préoxygénation au masque, a consisté en l'administration séquentielle de fentanyl 1 à $1,5 \mu \mathrm{g} \cdot \mathrm{kg}^{-1}$, puis deux minutes plus tard de thiopentone 3 à $5 \mathrm{mg} \cdot \mathrm{kg}^{-1}$ ainsi que de vécuronium $0,1 \mathrm{mg} \cdot \mathrm{kg}^{-1}$ pour faciliter l'intubation de la trachée. Le degré de relaxation musculaire a été surveillé au moyen d'un stimulateur neuro-musculaire. L'anesthésie a été maintenue par l'inhalation d'un mélange protoxyde d'azote - oxygène $(2: 1)$, associé à l'isoflurane (concentration inspirée: 0,5 à $1,5 \%$ ) et par des injections répétées de fentanyl 50 à $150 \mu \mathrm{g}$ selon les besoins, estimés en fonction de l'évolution clinique et des paramètres hémodynamiques des patients en réponses aux stimulations chirurgicales. La ventilation mécanique normocapnique a été assurée au moyen du respirateur Ohmeda $700{ }^{\circledR}$ et contrôlée par un capnographe $\left(\mathrm{CO}_{2}\right.$ Monitor $\left.5210 \mathrm{Ohmeda}^{\circledR}\right)$. Un garrot pneumatique a été installé à la racine du membre inférieur opéré puis, après exsanguination du membre au moyen d'une bande d'Esmarch, il a été gonflé à $300-350 \mathrm{mmHg}$. A la fin de lintervention chirurgicale le garrot pneumatique a été relâché. Après réveil et extubation, les patients ont été transférés en salle de réveil afin de surveiller leurs fonctions vitales.

\section{Variables mesurées}

La tension artérielle a été mesurée toutes les 2,5 minutes dès le début de l'induction. L'analyse porte sur les valeurs enregistrées à des temps particuliers: 1) immédiatement avant l'induction; 2) une minute après l'intubation; 3) cinq minutes après l'intubation; 4) immédiatement avant le gonflage du garrot; 5) immédiatement avant le relâche- 
TABLEAU I Paramètres démographiques (moyenne \pm SD)

\begin{tabular}{lll}
\hline & $\begin{array}{l}\text { Groupe } \\
\text { captopril } \\
n=10\end{array}$ & $\begin{array}{l}\text { Groupe } \\
\text { placebo } \\
n=10\end{array}$ \\
\hline Âge (années) & $37,4 \pm 13,4$ & $41,1 \pm 12,2$ \\
Poids (kg) & $77,8 \pm 7,1$ & $71,7 \pm 9,0$ \\
Taille (cm) & $179,1 \pm 5,0$ & $170,3 \pm 5,6^{*}$ \\
Sexe (M/F) & $(9 / 1)$ & $(6 / 4)$ \\
ASA (1/11) & $(10 / 0)$ & $(9 / 1)$ \\
TAS (mmHg) & $118,7 \pm 8,2$ & $124,9 \pm 15,4$ \\
TAD (mmHg) & $73,7 \pm 8,5$ & $75,7 \pm 7,4$ \\
TAM (mmHg) & $88,7 \pm 7,4$ & $92,1 \pm 9,4$ \\
FC (p/min) & $67,4 \pm 6,0$ & $64,9 \pm 6,0$ \\
\hline
\end{tabular}

$* P<0,01$.

TAS, TAD et TAM = tension artérielle systolique, diastolique et moyenne, respectivement.

$\mathrm{FC}=$ fréquence cardiaque.

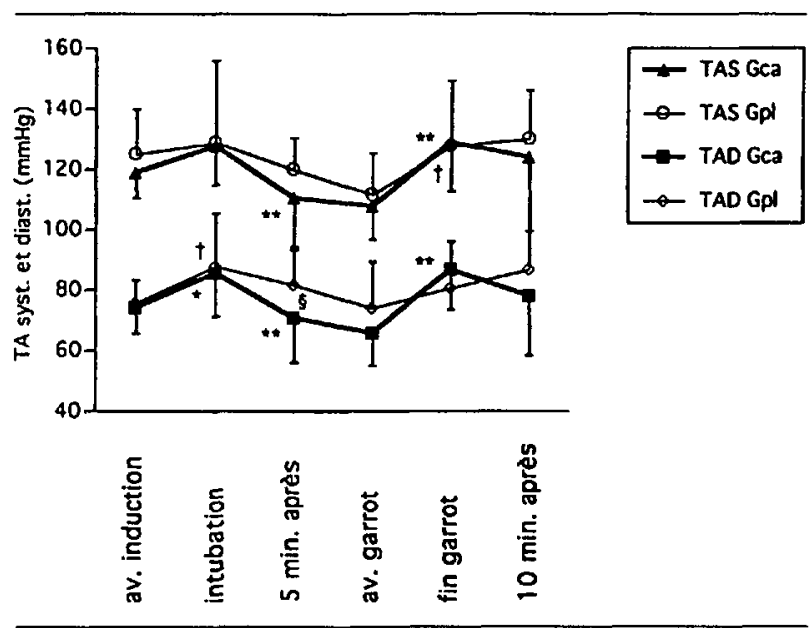

FIGURE 1 Évolution des TA systolique et diastolique au cours de l'intervention dans chaque groupe. ${ }^{*}(P<0,05)$ et ${ }^{* *}(P<0,01)$ indiquent une variation significative entre deux mesures successives dans le groupe captopril; $\dagger(P<0,05)$ et $\ddagger(P<0,01)$ ont la même signification pour le groupe placebo. §̧indique une différence significative $(P<0,05)$ entre les deux groupes à un temps particulier.

ment du garrot; et 6) dix minutes après le relâchement, ceci correspondant parfois au moment de l'extubation.

La TA, la fréquence cardiaque, la saturation artérielle périphérique en $\mathrm{O}_{2}\left(\mathrm{SpO}_{2}\right)$, la $\mathrm{FeCO}_{2}$, la $\mathrm{FiN}_{2} \mathrm{O}$, les fractions inspirée et expirée d'isoflurane ont été enregistrées et imprimées en temps réels au moyen d'une imprimante (HP Think Jet ${ }^{\circledR}$ ) directement reliée à l'appareil de monitorage (Datex Cardiocap ${ }^{\circledR}$ et Capnomac ${ }^{\circledR}$ ). La quantité totale d'opiacés a été comptabilisée.

\section{Analyse statistique}

Tous les résultats sont exprimés en valeur moyenne plus ou moins la déviation standard. La recherche d'éventuelles différences entre les deux groupes pour la répar-
TABLEAU II Tensions artérielles moyennes aux temps étudiés $(\mathrm{mmHg} \pm \mathrm{SD})$

\begin{tabular}{lcc}
\hline & $\begin{array}{l}\text { Groupe } \\
\text { captopril } \\
n=10\end{array}$ & $\begin{array}{l}\text { Groupe } \\
\text { placebo } \\
n=10\end{array}$ \\
\hline Av. induction & $88,7 \pm 7,4$ & $92,1 \pm 9,4$ \\
Intubation & $100,1 \pm 13,5^{*}$ & $101,7 \pm 20,4$ \\
5 min. après & $84 \pm 14,8^{* *}$ & $94,4 \pm 10,5 \dagger$ \\
Av. garrot & $80,2 \pm 10,1$ & $86,4 \pm 14,8^{*}$ \\
Fin garrot & $100,6 \pm 14,0^{* *}$ & $97,2 \pm 17,5$ \\
Extubation & $93,6 \pm 20,7$ & $101,4 \pm 12,8$ \\
\hline
\end{tabular}

$\dagger P<0,01$ entre les deux groupes.

${ }^{*} P<0,05$ et ${ }^{* *} P<0,01$ entre deux mesures successives, au sein d'un groupe.

tition des sexes et des classes ASA a été effectuée au moyen du Chi carré. Les comparaisons entre les groupes ont été effectuées avec le test de Mann-Whitney pour les données démographiques, les durées d'anesthésie générale et de gonflage de garrot, les concentrations expirées d'isoflurane, les quantités administrées de fentanyl, la tension artérielle et la fréquence cardiaque.

L'étude statistique des variations des valeurs hémodynamiques à l'intérieur d'un même groupe ont été étudiées à l'aide d'un ANOVA.

\section{Résultats}

Les dix patients du groupe captopril et huit patients du groupe placebo ont subi une arthroscopie du genou, un patient a eu une ablation de matériel d'ostéosynthèse et le dernier patient a eu une réduction et ostéosynthèse d'une fracture du platetau tibial. Le Tableau I résume les caractéristiques démographiques des deux groupes. Il n'y a pas de différence significative entre les deux groupes, si ce n'est pour la taille, ceci étant probablement dû au plus grand nombre de femmes dans le groupe placebo. La tension artérielle et le pouls préopératoire, mesurés avant l'induction, ne sont pas différents dans les deux groupes. La durée de l'anesthésie $(98,8 \pm 33,9 \mathrm{~min}$ vs $77,6 \pm 18,1 \mathrm{~min})$, la durée de l'application du garrot $(60,1$ $\pm 18,3 \mathrm{~min}$ vs $53,5 \pm 18,8 \mathrm{~min}$ ), la dose totale de fentanyl administrée $\left(2,59 \pm 0,56 \mu \mathrm{g} \cdot \mathrm{kg} \cdot \mathrm{h}^{-1}\right.$ vs $2,8 \pm 0,46$ $\mu \mathrm{g} \cdot \mathrm{kg} \cdot \mathrm{h}^{-1}$ ) et la moyenne de la fraction expirée d'isoflurane $(0,315 \pm 0,062$ vol\% vs $0,32 \pm 0,067$ vol\%) ne diffèrent pas de manière significative entre les groupes placebo et captopril respectivement.

Durant toute l'anesthésie, les tensions artérielles évoluent dans les deux groupes selon des mêmes tendances (Figure 1 et Tableau II). A un seul temps de l'intervention, cinq minutes après l'intubation, les moyennes des tensions artérielles (TA) diastoliques et des TA moyennes sont significativement plus basses dans le groupe captopril. Par ailleurs, au sein de chaque groupe, il y a des variations 
significatives entre deux périodes de mesures consécutives, plus fréquemment dans le groupe captopril que dans le groupe placebo.

La fréquence cardiaque (Figure 2) évolue également dans les deux groupes selon les mêmes tendances, par contre, à aucune période on ne trouve de différence significative entre les deux groupes.

\section{Discussion}

Les bénéfices théoriques liés à l'administration d'un inhibiteur de l'enzyme de conversion lors d'anesthésie générale comprennent: 1) l'atténuation de l'élévation tensionnelle survenant pendant la période de gonflage d'un garrot pneumatique; 2) une diminution de la dose totale d'agents anesthésiques (fentanyl, isoflurane) utilisés pendant la période de gonflage du garrot; 3) Yates et Hunter ${ }^{12}$ montrent que des patients prétraités avec de l'énalapril, présentent une plus grande stabilité hémodynamique lors de stimulations (intubation, incision) sous anesthésie générale. Cette stabilité se traduit essentiellement par une atténuation des variations tensionnelles.

Dans le groupe captopril (Gca), l'évolution des variations de pression artérielle systémique au cours de l'opération ne diffère pas de façon cliniquement significative de celle observée dans le groupe placebo $(\mathrm{Gpl})$. En particulier, les TA augmentent significativement pendant la durée de gonflage du garrot pneumatique dans les deux groupes. Le prétraitement par un inhibiteur de l'enzyme de conversion n'a donc pas permis de prévenir cette augmentation tensionnelle. Au contraire, celle-ci est plus significative dans le groupe captopril que dans le groupe placebo. Une étude ${ }^{10}$ ayant montré qu'une dose de 25 mg per os de captopril était suffisante pour inhiber l'axe rénine-angiotensine, le dosage de $50 \mathrm{mg}$ est certainement suffisant. De ce fait, on peut conclure que l'axe rénineangiotensine nintervient pas, ou peu dans le phénomène de l'apparition de l'EPA induite par un garrot pneumatique. L'inhibition de l'axe rénine-angiotensine, démontrée par l'augmentation de l'activité de la rénine plasmatique (ARP) n'a pas été documentée par un dosage car il s'agit d'un effet bien documenté par d'autres groupes. ${ }^{10,13}$ De plus, des évidences expérimentales, suggèrent que la TA systémique est plus liée à la concentration de rénine tissulaire qu'à l'ARP. ${ }^{14}$

La notion d'HTA associée à l'application d'un garrot pneumatique est essentiellement descriptive. Définie tantôt par une augmentation de $30 \%$ de la tension artérielle de base, ${ }^{2,4.5}$ tantôt par une augmentation statistiquement significative de la tension artérielle, ${ }^{3}$ les valeurs de la tension artérielle ne sont que rarement précisées. Dès lors, on peut avoir soit une élévation de la tension artérielle soit une HTA vraie. Le fait est que le mécanisme de cette modification hémodynamique reste inconnu.

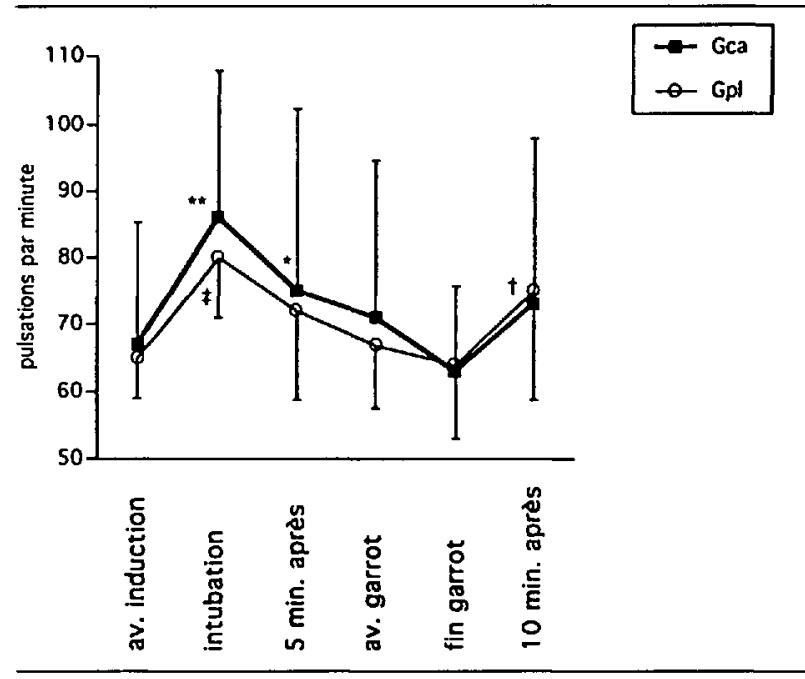

FIGURE 2 Evolution de la fréquence cardiaque au cours de l'intervention dans chaque groupe. ${ }^{*}(P<0,05)$ et ${ }^{* *}(P<0,01)$ indiquent une variation significative entre deux mesures successives dans le groupe captopril; $\dagger(P<0,05)$ et $\ddagger(P<0,01)$ ont la même signification pour le groupe placebo.

Dans notre étude, les valeurs tensionnelles maximales atteintes durant la période de gonflage du garrot ne dépassent que rarement les valeurs reconnues pour définir une HTA. En moyenne, à la fin de la période de gonflage $\left(T_{5}\right)$ du garrot, les TA systoliques et diastoliques ont été de 128/86 et de 128/81 dans le Gca et dans le Gpl, respectivement. De plus, seul deux patients du Gca et un patient du $\mathrm{Gpl}$ ont des valeurs tensionnelles au $T_{S}$ pouvant faire nécessiter un traitement. Un seul auteur ${ }^{4}$ signale des TA systoliques dépassant $190 \mathrm{mmHg}$ avec un cas d'infarctus du myocarde fatal. Il a pu montrer que les facteurs de risque associés à cette EPA étaient l'âge, la cardiomégalie à la radiographie thoracique, l'anesthésie générale associant le protoxyde d'azote et des opiacés ainsi que la durée de gonflage du garrot. A linverse, l'EPA induite par le garrot ne semble pas être favorisée par une HTA préexistante ou une cardiopathie ischémique. ${ }^{2,4}$ De ce fait, on peut conclure que, pour une population ne présentant aucun de ces facteurs de risque, un traitement ou une prévention de cette augmentation des valeurs hémodynamiques ne se justifie pas. Une bonne anesthésie balancée associant $\mathrm{N}_{2} \mathrm{O}$, isoflurane et opiacés devrait suffire pour maintenir les TA dans des limites acceptables. Il faut noter qu'il n'y a pas eu d'adjonction d'hypotenseurs dans notre étude et qu'en particulier la quantité d'isoflurane administrée dans les deux groupes a été identique. La plus grande stabilité hémodynamique suggérée par d'autres groupes, ${ }^{12}$ n'a pas été retrouvée dans notre collectif où le profil tensionnel a varié davantage chez les malades qui avaient été prétraités avec du captopril. En effet, la variation du profil ten- 
sionnel au cours de l'anesthésie générale a été identique dans les deux groupes mais ces variations ont été plus marquées dans le groupe captopril: la moyenne des TA moyennes (Tableau II) varie significativement à trois temps différents de l'intervention, alors que dans le groupe contrôle, une seule variation significative est relevée.

En conclusion, le prétraitement par du captopril, n'a pas permis de prévenir l'élévation tensionnelle survenant sous anesthésie générale lors de chirurgie du membre inférieur pratiquée avec un garrot pneumatique. Mais les valeurs hémodynamiques mesurées, tant dans le groupe placebo que dans le groupe captopril, démontrent qu'une telle prévention n'est pas nécessaire.

\section{Références}

1 Bradford EMW. Haemodynamic changes associated with the application of lower limb tourniquets. Anaesthesia 1969; 24: 190-7.

2 Kaufman RD, Walts LF. Tourniquet-induced hypertension. Br J Anaesth 1982; 54: 333-6.

3 Rocco AG, Concepcion MA, Murray E, Covino BG. Effect of general and regional anesthesia on tourniquet induced hypertension. Anesthesiology 1984; 61: A195.

4 Valli $H$, Rosenberg PH, Kyttä J, Nurminen $M$. Arterial hypertension associated with the use of a tourniquet with either general or regional anaesthesia. Acta Anaesthesiol Scand 1987; 31: 279-83.

5 Valli $H$, Rosenberg $P H$. Effects of three anaesthesia methods on haemodynamic responses connected with the use of thigh tourniquet in orthopaedic patients. Acta Anaesthesiol Scand 1985; 29: 142-7.

6 De Jong RH, Nace RA. Nerve impulse conduction and cutaneous receptor responses during general anesthesia. Anesthesiology 1967; 28: 851-5.

7 Strichartz $G$, Zimmerman $M$. An explanation for pain originating from tourniquets during regional anesthesia. ASRA 9th Annual Meeting Abstracts. Reg Anaesth 1984; 9: 44-5.

8 Erard PY, Gardaz JP, Nussberger J, et al. Effets de la clonidine sur l'hypertension artérielle systémique sous garrot lors d'anesthésie générale. Ann Fr Anesth Réanim 1989; 8 : R198.

9 Vidt DB, Bravo EL, Fouad FM. Captopril. N Engl J Med 1982; 306: 214-9.

10 Pasch Th, Kleierl-Lindner C, Götz H, Pichl J. Untersuchungen uber den uberschiessenden blutdruckanstieg nach Kontrollierter Hypotension und seine Verhütung durch captopril. Anaesthesist 1986; 35: 66-72.

11 Edwards CRW, Padfield PL. Angiotensin-converting enzyme inhibitors: past, present, and bright future. Lancet 1985; 1: 30-4.

12 Yates $A P$, Hunter $D N$. Anaesthesia and angiotensinconverting enzyme inhibitors. The effect of enalapril on peri-operative cardiovascular stability. Anaesthesia 1988; 43 : 935-8.

13 Woodside J Jr., Garner L, Bedford RF, et al. Captopril reduces the dose requirement for sodium nitroprusside induced hypotension. Anesthesiology 1984; 60: 413-7.

14 Dzau VJ. Implications of local angiotensin production in cardiovascular physiology and pharmacology. Am J Cardiol 1987; 59: 59A-65A. 